\title{
In vitro antitumor effect of sodium butyrate and zoledronic acid combined with traditional chemotherapeutic drugs: A paradigm of synergistic molecular targeting in the treatment of Ewing sarcoma
}

\author{
MICHEL PINHEIRO DOS SANTOS ${ }^{1,3}$, CAROLINE BRUNETTO DE FARIAS ${ }^{1,2,4}$, \\ RAFAEL ROESLER ${ }^{1,4,5}$, ALGEMIR LUNARDI BRUNETTO ${ }^{1,2,4}$ and ANA LUCIA ABUJAMRA ${ }^{1-4}$
}

${ }^{1}$ Cancer Research Laboratory, University Hospital Research Center (CPE-HCPA), Federal University of Rio Grande do Sul;
${ }^{2}$ Children's Cancer Institute and Pediatric Oncology Unit, Federal University Hospital (HCPA); ${ }^{3}$ Medical Sciences Program,
School of Medicine, Federal University of Rio Grande do Sul; ${ }^{4}$ National Institute for Translational Medicine (INCT-TM);
${ }^{5}$ Laboratory of Neuropharmacology and Neural Tumor Biology, Department of Pharmacology,
Institute for Basic Health Sciences, Federal University of Rio Grande do Sul, Porto Alegre, RS, Brazil

Received September 24, 2013; Accepted October 29, 2013

DOI: $10.3892 /$ or.2013.2907

\begin{abstract}
Histone deacetylase inhibitors and bisphosphonates have a promising future in the treatment of cancer as targeted anticancer drugs, particularly when used together or in combination with other cytotoxic agents. However, the effects of these combined treatments have not yet been systematically evaluated in Ewing sarcoma. The in vitro effects on cellular proliferation, viability and survival were investigated in two Ewing sarcoma cell lines, SK-ES-1 and RD-ES. The cell lines were treated with sodium butyrate, a histone deacetylase inhibitor and zoledronic acid, a bisphosphonate, alone, together or in combination with chemotherapeutic drugs recommended for clinical treatment of Ewing sarcoma. The data demonstrated that the combination of sodium butyrate and zoledronic acid had a synergistic cytotoxic effect at $72 \mathrm{~h}$ following treatment, persisting for 10-14 days post-treatment, in both cell lines tested. All combinations between sodium butyrate or zoledronic acid and the traditional antineoplastic drugs (doxorubicin, etoposide and vincristine) demonstrated a synergistic cytotoxic effect at $72 \mathrm{~h}$ in SK-ES-1 and RD-ES cells, except for the combinations of sodium butyrate with vincristine and of zoledronic acid with doxorubicin, which showed only an additive effect in RD-ES cell lines as compared to each agent alone. These acute effects observed in both Ewing sarcoma cell lines were confirmed by the clonogenic assay. The present
\end{abstract}

Correspondence to: Professor Ana Lucia Abujamra, Children's Cancer Institute and Pediatric Oncology Unit, Federal University Hospital (HCPA), Rua Ramiro Barcelos 2350, $3^{\circ}$ Leste, Porto Alegre, RS 90035-903, Brazil

E-mail: aabujamra@hcpa.ufrgs.br

Key words: Ewing sarcoma, sodium butyrate, zoledronic acid, adjuvant therapy, chemotherapy, synergistic effect data suggest that combining histone deacetylase inhibitors and bisphosphonates with traditional chemotherapeutic drugs is a promising therapeutic strategy for the treatment of Ewing sarcoma, and provides a basis for further studies in this field.

\section{Introduction}

Ewing sarcoma (ES), often referred to as Ewing's sarcoma family tumors (ESFTs), is a peripheral primitive neuroectodermal tumor (PNET). ES is the second most common solid bone and soft tissue malignancy of children and young adults following osteosarcoma, and accounts for $~ 3 \%$ of all malignancies in pediatric patients (1-3). This disease most commonly occurs in the second decade of life, and $~ 55 \%$ of affected individuals are male (4). It is associated in $85 \%$ of cases with the $(11 ; 22)(\mathrm{q} 24: \mathrm{q} 12)$ chromosomal translocation that generates fusion of the 50 first segments of the EWS gene with the 30 last segments of the FLI-1 gene of the ETS family. The resulting EWS-FLI-1 fusion protein is believed to behave as an aberrant transcriptional activator that contributes to ESFT development by altering the expression of its target genes in a permissive cellular environment (5). Current treatments comprise neoadjuvant chemotherapy and local surgical resection with limb salvage, followed by adjuvant chemotherapy with or without radiotherapy. Most combination chemotherapeutic regimens are based on alkylating agents, primarily ifosfamide and cyclophosphamide and anthracyclines, supplemented with vinca alkaloids, actinomycin D and etoposide (6). Before the era of chemotherapy, the survival rate of ES patients was $<10 \%$ (7). The current use of an aggressive multimodal therapy has improved long-term survival rates of patients with localized disease in most clinical centers by $50-70 \%$. However, patients with clinically detectable metastases at diagnosis, who do not respond to therapy or with disease relapse, have a significantly poorer prognosis $(20 \%)$. In addition, $30-40 \%$ of patients with ES experience disease relapse. They have a particularly 
dismal prognosis, with a survival likelihood that does not exceed $20 \%(3,5,8)$. Moreover, traditional chemotherapeutic agents are often used in high doses, causing several toxic side-effects, including neurotoxicity in the case of vincristine, hematological side-effects in the case of ifosfamide or etoposide or cardiotoxicity in the case of doxorubicin (9-12). For these reasons, efforts to identify new therapies are still warranted (6).

Epigenetic alterations, such as histone deacetylation, are known to contribute to cellular transformation by silencing critical genes. Histone deacetylase inhibitors (HDACIs or HDIs), such as sodium butyrate $(\mathrm{NaB})$, represent a potentially novel class of antineoplastic agents with the potential to act as adjuvants, since they promote or enhance a variety of distinct anticancer mechanisms, including apoptosis, cell cycle arrest, cellular differentiation, immune stimulatory and antiangiogenic activities. A number of HDIs are currently under clinical trials, owing to their ability to revert some of the aberrant epigenetic states associated with cancer. These studies have shown that histone hyperacetylation can be safely achieved in humans, and that treatment with such agents is plausible (13-15). The use of HDIs alone or in association with other molecules, such as traditional chemotherapeutic drugs, could be a promising alternative for the treatment of cancer patients in the future (15-18).

The bisphosphonates (BPs) are generally well-known for their antiresorptive effects on bone metabolism, providing a reductive effect on the morbidity of skeletal metastasis of different types of tumors, and also serving as a primary therapeutic option for malignant hypercalcemia. Zoledronic acid (ZA), the most potent member of the nitrogen-containing bisphosphonate family (N-BPs), inhibits osteoclastic resorptive activity partly through inhibition of farnesyl-diphosphate synthase and protein prenylation. However, far beyond their effect on bone turnover, bisphosphonates have pleiotropic biological effects: they can mediate growth factor release, cell adhesion, antiangiogenic effects and apoptosis in various types of tumors (19). Recent in vitro and in vivo studies suggest that bisphosphonates exhibit antitumor activity, especially when associated with other cytotoxic agents, such as those employed in traditional chemotherapy (20-22).

HDIs and bisphosphonates have a promising future in the treatment of cancer as targeted anticancer agents, especially when used together or in combination with other molecules, such as traditional anticancer drugs. However in ES, the possible effects of co-administering HDIs and bishosphonates, and of associating HDIs or bisphosphonates with traditional anticancer drugs have not yet been systematically investigated. In the present study, we evaluated the in vitro cytotoxic effects elicited by the co-treatment with $\mathrm{NaB}$ and $\mathrm{ZA}$ alone or in combination with three anticancer drugs strongly recommended for the treatment of ES (doxorubicin, etoposide and vincristine) in two human ES cell lines, SK-ES-1 and RD-ES.

\section{Materials and methods}

Cell lines and reagents. The human Ewing sarcoma cell lines SK-ES-1 and RD-ES were obtained from the American Type Culture Collection (ATCC; Rockville, MD, USA) and grown in RPMI-1640 medium (Gibco-BRL, Carlsbad, CA,
USA), containing $0.1 \%$ Fungizone ${ }^{\circledR}$ (250 mg/kg; Invitrogen Life Technologies, São Paulo, Brazil), 100 U/l gentamicin (4 mg/ml; Nova Pharma, Brazil) and $10 \%$ (SK-ES-1) or $15 \%$ (RD-ES) fetal bovine serum (FBS; Sorali, Campo Grande, Brazil), at $37^{\circ} \mathrm{C}$ in a humidified incubator under $5 \% \mathrm{CO}_{2}$. Sodium butyrate $(\mathrm{NaB})$ and trypan blue were obtained from Sigma-Aldrich (St. Louis, MO, USA); zoledronic acid (ZA), vincristine (VCR) and doxorubicin (Doxo) were obtained from Faulding/LIBBS (São Paulo, Brazil); and etoposide (VP-16) was purchased from Bristol-Myers Squibb (São Paulo, Brazil).

Cellular proliferation and viability assay. The cells were seeded at a density of $2.5 \times 10^{4}$ cells/well (SK-ES-1) and $3.5 \times 10^{4}$ cells/well (RD-ES) in 24-well plates and allowed to grow for $24 \mathrm{~h}$. The medium was replaced and different doses of NaB, ZA, VCR, VP-16 and Doxo, alone or in combination, were added to the culture medium. To measure cellular proliferation, the medium was removed $72 \mathrm{~h}$ following the treatments, cells were washed with Hanks' balanced salt solution (Invitrogen Life Technologies) and were detached with $100 \mu \mathrm{l}$ of $0.25 \%$ trypsin solution. To inactivate trypsin, $100 \mu \mathrm{l}$ of complete medium was added, and $10 \mu \mathrm{l}$ of the resulting cellular suspension was mixed with $10 \mu 1$ trypan blue $(0.4 \%)$. Cells were then manually counted in a hemocytometer to determine the ratio of dead to viable cells $(23,24)$. The mean of triplicate experiments for each dose was used to calculate the $\mathrm{IC}_{50}$ and the combination index (CI) values. The results were confirmed by an MTT assay (25).

Colony formation assay(clonogenic survival assay). Following counting, SK-ES-1 and RD-ES cells were seeded into 6-well plates without treatment (1,000 cells/well). After incubation for 10-14 days, the cells were fixed with $70 \%$ ethanol and counterstained with $0.5 \%$ crystal violet (26).

Optimized digital counting of colonies for the clonogenic assays. To analyze the clonogenic assay data, colonies were digitalized and an optimized digital colony counting was performed using ImageJ software (version 1.37 for Windows) (27). The method developed by Cai et al (27) was validated according to the experimental conditions. After drying the crystal violet stain, images from each plate were obtained using a desktop scanner (Lexmark X342n XL). Each plate was placed at the same position on the light table by aligning it with the center of the exposed preview light window. In order to separate colonies from the dark grey background, each plate was scanned in color or grey-scale mode. The scanned images were saved as 16-bit tiff files with a resolution setting of 600 dots per inch (DPI) and were analyzed using the ImageJ software as follows: First, image files of the same plate were opened, then the background was subtracted ('Process $\backslash$ Subtract Background...') to correct for unevenness of transmission light. The default setting for a rolling ball radius of 15.0 pixels was chosen and the light background was checked. Following background subtraction, the images were converted into 8-bit grey scale images. Second, the macro 'Make_ovalscan.txt' was run, resulting in the appearance of the region of interest in the shape of a circle that corresponds to a circular well area. Next, colony counting was performed by setting the threshold values ('Image \Adjust\Threshold...'), 
the measurements ('Analyze $\backslash$ Set Measurements...') and the parameters that were measured and displayed: the area, the mean grey value, the standard deviation (SD), the integrated density and the display label. The colonies were analyzed ('Analyze\Analyze Particles...') with a range of predefined values for particle (colony) size ('5-Infinity') and circularity ('0.30-1.00'). The effects of drugs on treated colonies were expressed as the surviving fraction of the colonies (SF) and the average size of the colonies (AS) evaluated with the following formulas:

i) $\mathrm{SF}=\frac{\text { Number of colonies in treated cells } \mathrm{x} 100}{\text { Number of colonies in control }}$

ii) $\mathrm{AS}=\frac{\text { Total area of colonies in treated cells }}{\text { Number of colonies in treated cells }}$

To confirm the results, the colonies in each well were also manually counted using light microscopy. Only colonies consisting of at least 50 cells were counted.

Statistical analysis. Results are representative of three independent experiments performed in triplicates, and are expressed as mean values \pm standard deviation (SD) of independent experiments. Data were analyzed by a one-way analysis of variance (ANOVA), followed by the Tukey-Kramer test, using the SPSS program, version 16.0. P-values $(\mathrm{P})<0.05$ were considered to indicate statistically significant differences.

Median dose-effect analysis. The combination index, a measure of synergism and antagonism, is calculated based on the method described by Chou and Talalay along with the release of the computer software CalcuSyn (Biosoft, Ferguson, MO, USA), designed to assess drug interactions. This method takes into account both the potency of each drug or combination of drugs and the shape of the dose-effect curve. The CalcuSyn software version 2.11 for Windows was used to calculate the CI, with synergy, additivity and antagonism defined as $\mathrm{CI}<0.9, \mathrm{CI}=0.9-1.1$ and $\mathrm{CI}>1.1$ respectively, and $\mathrm{CI} \leq 0.3$ defined as strong synergy. For this analysis, we chose clinically achievable concentrations of $\mathrm{NaB}, \mathrm{ZA}, \mathrm{VCR}$, Doxo and VP-16 that were below the $\mathrm{IC}_{50}$ values $(28,29)$.

\section{Results}

A time-and dose-dependent decrease in the proliferation of ES cell lines in response to sodium butyrate, zoledronic acid or traditional antineoplastic drugs. To evaluate the cytotoxic effects of sodium butyrate on the growth of Ewing sarcoma cells, SK-ES-1 and RD-ES cell lines were exposed to increasing concentrations of $\mathrm{NaB}(0.1-10 \mathrm{mM})$ for 24,48 and $72 \mathrm{~h}$, and trypan blue counting assays were performed. There was an 8 , 27 and $55 \%$ decrease in the viability of SK-ES-1 cells exposed to $0.1,0.5$ and $1 \mathrm{mM}$ of $\mathrm{NaB}$, respectively, as compared to the untreated controls at $72 \mathrm{~h}$ (Fig. 1A). In addition, there was a 11, 30 and $75 \%$ decrease in the viability of RD-ES cells exposed to $0.5,0.75$ and $3 \mathrm{mM}$ of $\mathrm{NaB}$, respectively, when compared to the untreated controls at $72 \mathrm{~h}$ (Fig. 1B). The highest cytotoxicity was observed at $72 \mathrm{~h}$ and $\mathrm{IC}_{50}$ values of $\mathrm{NaB}$ in SK-ES-1 and RD-ES cells were estimated at 0.77 and $1.08 \mathrm{mM}$, respectively. The effect of zoledronic acid on SK-ES-1 and
RD-ES cells was evaluated at increasing concentrations of ZA (1-200 $\mu \mathrm{M})$ for 24,48 and $72 \mathrm{~h}$, and cell proliferation assays were performed. There was a 15, 45 and $80 \%$ decrease in the viability of SK-ES-1 cells exposed to 10,25 and $100 \mu \mathrm{M}$ of ZA, respectively, when compared to the untreated controls at $72 \mathrm{~h}$ (Fig. 1C). In addition, there was a 7, 36 and $84 \%$ decrease in the cell viability of RD-ES cells exposed to 10,50 and $200 \mu \mathrm{M}$ of ZA, respectively, when compared to the suntreated controls at $72 \mathrm{~h}$ (Fig. 1D). The $\mathrm{IC}_{50}$ value of zoledronic acid was $34.31 \mu \mathrm{M}$ for SK-ES-1 cells and $67.45 \mu \mathrm{M}$ for RD-ES cells.

We also examined the effects of the traditional chemotherapeutic agents doxorubicin, etoposide and vincristine on SK-ES-1 and RD-ES cell lines. The cells were exposed to increasing concentrations of Doxo (0.1-200 nM), VP-16 (0.01-10 $\mu \mathrm{M})$ and VCR (0.1-10 $\mathrm{nM})$ for 24, 48 and $72 \mathrm{~h}$, and cell proliferation assays were performed. There was a 25,40 and $79 \%$ decrease in cell viability of SK-ES-1 cells exposed to 10,15 and $50 \mathrm{nM}$ of Doxo, respectively, when compared to the untreated controls at $72 \mathrm{~h}$ (Fig. 1E). In addition, there was a 10, 40 and $90 \%$ decrease in the viability of RD-ES cells exposed to 10,30 and $100 \mathrm{nM}$ of Doxo, respectively, as compared to the untreated controls at $72 \mathrm{~h}$ (Fig. 1F). The highest cytotoxicity was observed at $72 \mathrm{~h}$ and the $\mathrm{IC}_{50}$ values of Doxo in SK-ES-1 and RD-ES cells were estimated at 19.83 and $32.48 \mathrm{nM}$, respectively. There was a 24, 44 and $91 \%$ decrease in the cell viability of SK-ES-1 cells exposed to $0.05,0.1$ and $0.5 \mu \mathrm{M}$ of VP-16, respectively, when compared to the untreated controls at $72 \mathrm{~h}$ (Fig. 1G). In addition, there was a 14,35 and $68 \%$ decrease in the viability of RD-ES cells exposed to $0.05,0.1$ and $0.6 \mu \mathrm{M}$ of VP-16, respectively, when compared to the untreated controls at $72 \mathrm{~h}$ (Fig. 1H). The highest cytotoxicity was observed at $72 \mathrm{~h}$ and $\mathrm{IC}_{50}$ values of VP-16 in SK-ES-1 and RD-ES cells were estimated at 0.11 and $0.17 \mu \mathrm{M}$, respectively. A 28, 67 and 95\% decrease in the cell viability of SK-ES-1 cells was found when they were exposed to $0.5,1$ and $5 \mathrm{nM}$ of VCR, respectively, when compared to the untreated controls at $72 \mathrm{~h}$ (Fig. 1I). In addition, there was a 25,68 and $82 \%$ decrease in viability of RD-ES cells exposed to 1,5 and $7.5 \mathrm{nM}$ of VCR, respectively, when compared to the untreated controls at $72 \mathrm{~h}$ (Fig. 1J). The highest cytotoxicity was observed at $72 \mathrm{~h}$ and the $\mathrm{IC}_{50}$ values of VCR in SK-ES-1 and RD-ES cells were estimated at 0.71 and $2.09 \mathrm{nM}$, respectively. All tested agents caused cytotoxicity in a time- and dose-dependent manner in SK-ES-1 and RD-ES cells (data not shown).

Sodium butyrate and zoledronic acid synergistically inhibit growth in the ES cell lines. To study the potentially synergistic/additive effects of $\mathrm{NaB}$ and ZA combined, SK-ES-1 and RD-ES cells were exposed to concentrations chosen as clinically achievable and below or at the $\mathrm{IC}_{50}$ values of each agent alone, and of their combination for $72 \mathrm{~h}$. Synergism or additivity was assessed via CI measures using the Calcusyn software. Results revealed synergistic toxicity in human Ewing sarcoma SK-ES-1 and RD-ES cells at $72 \mathrm{~h}$, as compared to each agent alone (Table I). The results indicated that $0.5 \mathrm{mM}$ $\mathrm{NaB}$ and $25 \mu \mathrm{M}$ ZA led to a 24 and $44 \%$ decrease in the cell viability of SK-ES-1 cells, respectively, but the combination of both led to a $72 \%$ decrease (Fig. 2A). In RD-ES cells, the results were similar: $0.75 \mathrm{mM} \mathrm{NaB}$ and $50 \mu \mathrm{M}$ ZA led to a 
A Sodium butyrate (mM) - SK-ES-1 cells

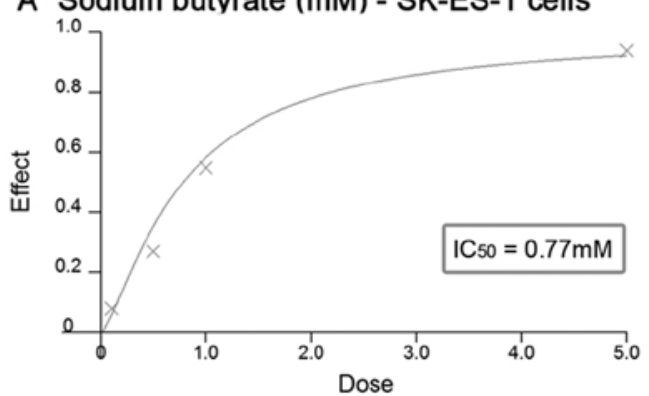

C Zoledronic acid $(\mu \mathrm{M})$ - SK-ES-1 cells

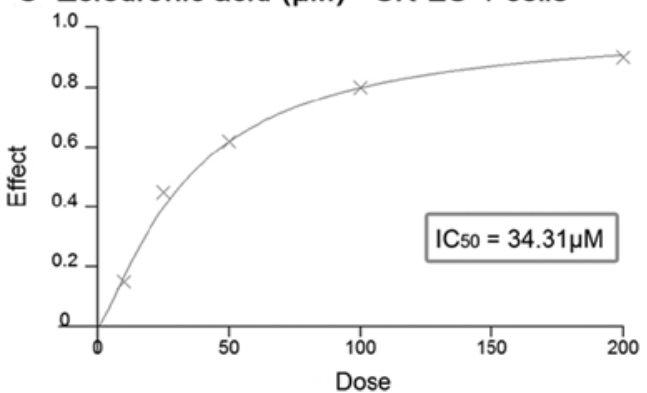

E Doxorubicin (nM) - SK-ES-1 cells

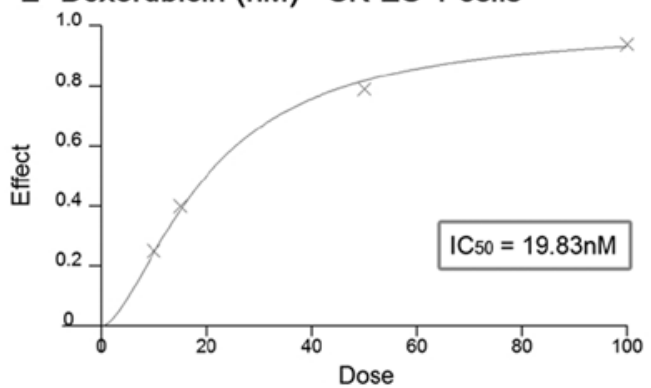

G Etoposide $(\mu \mathrm{M})$ - SK-ES-1 cells

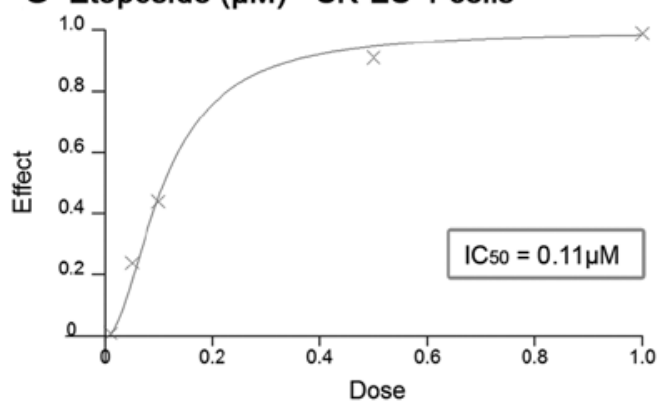

I Vincristine (nM) - SK-ES-1 cells

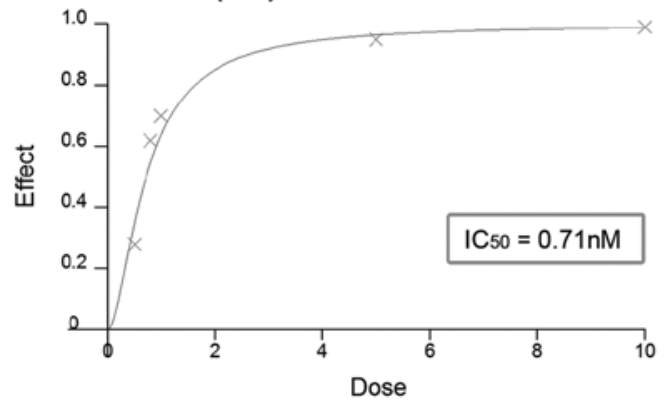

B Sodium butyrate (mM) - RD-ES cells

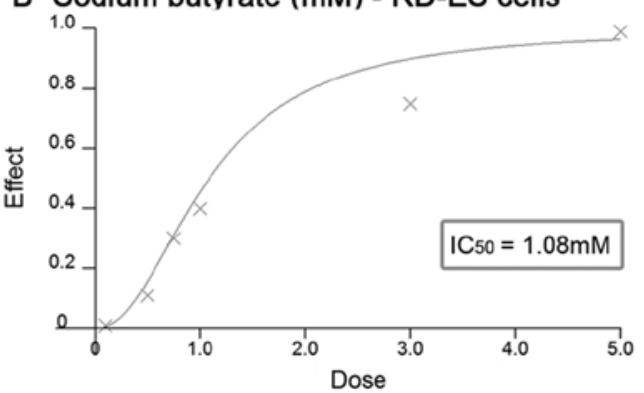

D Zoledronic acid $(\mu \mathrm{M})$ - RD-ES cells

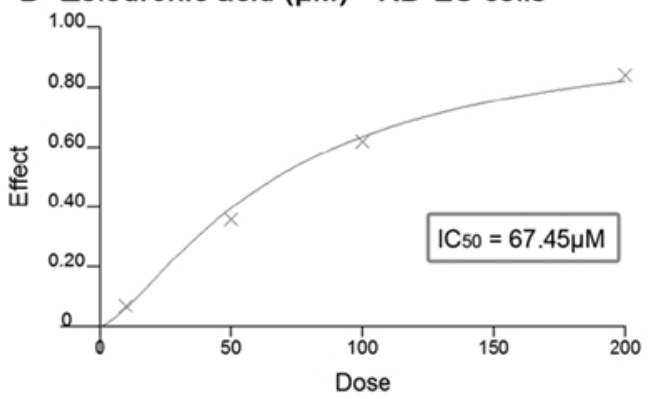

F Doxorubicin (nM) - RD-ES cells

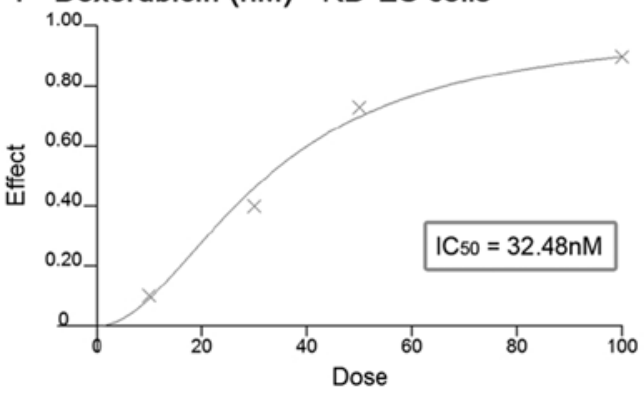

H Etoposide ( $\mu \mathrm{M})$ - RD-ES cells

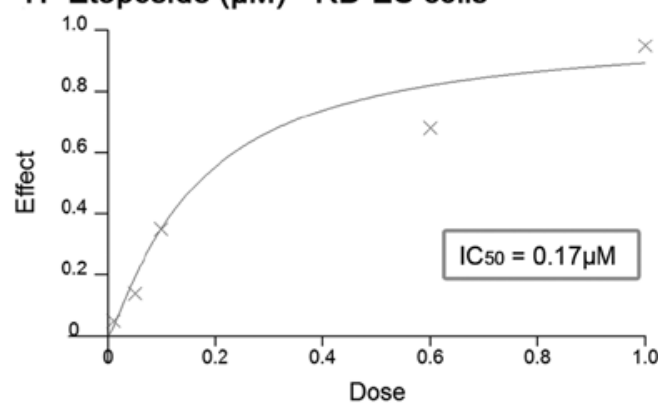

J Vincristine (nM) - RD-ES cells

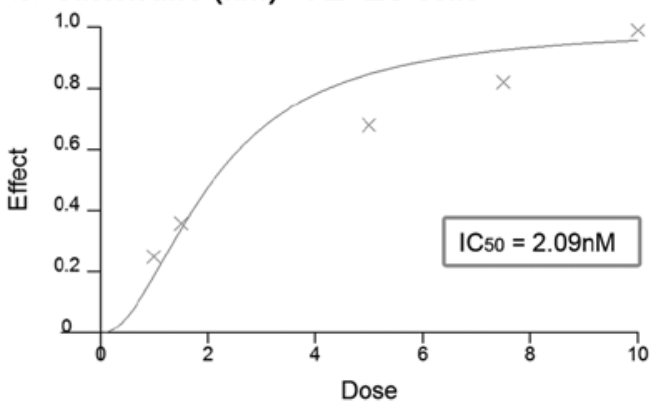

Figure 1. Dose-response study of the antitumor effect of sodium butyrate (A and B), zoledronic acid (C and D), doxorubicin (E and F), etoposide (G and $\mathrm{H})$ and vincristine (I and J) in human Ewing sarcoma (ES) SK-ES-1 and RD-ES cell lines, respectively. The $\mathrm{IC}_{50}$ concentration of each drug was determined with the trypan blue counting assay as described in Material and methods after a 72-h treatment of the cells with different drug concentrations. Cell viability was assessed in triplicates, in at least three independent experiments. Data are expressed as dose-effect curves: effect (fraction affected vs. control) represented on the y-axis, vs. dose (different doses of each agent tested), represented on the x-axis. The linear correlation coefficient (r) of the median-effect plot was $>0.90$ for all tested agents, ensuring accuracy of measurements and conformity to the mass-action. Positive controls corresponding to 100\% cell viability are denoted as ' 0 ' effect on the y-axis. 
Table I. Combination index values.

\begin{tabular}{|c|c|c|c|}
\hline Cell line & Concentration of drugs & CI value & Interpretation \\
\hline \multirow[t]{8}{*}{ SK-ES-1 } & $\mathrm{NaB}(0.5 \mathrm{mM})+\mathrm{ZA}(25 \mu \mathrm{M})$ & 0.687 & Synergism \\
\hline & $\mathrm{NaB}(0.5 \mathrm{mM})+\operatorname{Doxo}(15 \mathrm{nM})$ & 0.503 & Synergism \\
\hline & $\mathrm{NaB}(0.5 \mathrm{mM})+\mathrm{VP}-16(0.06 \mu \mathrm{M})$ & 0.532 & Synergism \\
\hline & $\mathrm{NaB}(0.5 \mathrm{mM})+\mathrm{VCR}(0.5 \mathrm{nM})$ & 0.533 & Synergism \\
\hline & $\mathrm{ZA}(25 \mu \mathrm{M})+\operatorname{Doxo}(15 \mathrm{nM})$ & 0.689 & Synergism \\
\hline & $\mathrm{ZA}(25 \mu \mathrm{M})+\mathrm{VP}-16(0.06 \mu \mathrm{M})$ & 0.729 & Synergism \\
\hline & $\mathrm{ZA}(25 \mu \mathrm{M})+\mathrm{VCR}(0.8 \mathrm{nM})$ & 0.769 & Synergism \\
\hline & $\mathrm{NaB}(0.5 \mathrm{mM})+\mathrm{ZA}(25 \mu \mathrm{M})+\mathrm{VP}-16(0.06 \mu \mathrm{M})$ & 0.287 & Strong synergism \\
\hline \multirow[t]{8}{*}{ RD-ES } & $\mathrm{NaB}(0.75 \mathrm{mM})+\mathrm{ZA}(50 \mu \mathrm{M})$ & 0.539 & Synergism \\
\hline & $\mathrm{NaB}(0.75 \mathrm{mM})+$ Doxo $(30 \mathrm{nM})$ & 0.491 & Synergism \\
\hline & $\mathrm{NaB}(0.75 \mathrm{mM})+\mathrm{VP}-16(0.08 \mu \mathrm{M})$ & 0.644 & Synergism \\
\hline & $\mathrm{NaB}(0.75 \mathrm{mM})+\mathrm{VCR}(1.5 \mathrm{nM})$ & 0.987 & Additivity \\
\hline & $\mathrm{ZA}(50 \mu \mathrm{M})+\operatorname{Doxo}(30 \mathrm{nM})$ & 0.922 & Additivity \\
\hline & $\mathrm{ZA}(50 \mu \mathrm{M})+\mathrm{VP}-16(0.08 \mu \mathrm{M})$ & 0.738 & Synergism \\
\hline & $\mathrm{ZA}(50 \mu \mathrm{M})+\mathrm{VCR}(1.5 \mathrm{nM})$ & 0.688 & Synergism \\
\hline & $\mathrm{NaB}(0.75 \mathrm{mM})+\mathrm{ZA}(50 \mu \mathrm{M})+\mathrm{VP}-16(0.08 \mu \mathrm{M})$ & 0.236 & Strong synergism \\
\hline
\end{tabular}

Combination index values (CI) of sodium butyrate (NaB), zoledronic acid (ZA), doxorubicin (Doxo), etoposide (VP-16) and vincristine (VCR) alone and in combination in SK-ES-1 and RD-ES cells. CI values were calculated from the cellular proliferation and viability assays. The data represent the mean of three independent experiments.

19 and $36 \%$ decrease in cell viability, respectively, while the combination of both led to a $73 \%$ decrease (Fig. 2A).

Sodium butyrate and traditional antineoplastic agents exhibit synergistic or additive effects on growth inhibition in the ES cell lines. These experiments were conducted in a similar fashion as described above for the $\mathrm{NaB}$ and ZA combination. Results showed synergistic toxicity or additive effects in human Ewing sarcoma SK-ES-1 and RD-ES cells at $72 \mathrm{~h}$, when $\mathrm{NaB}$ was combined with Doxo, VP-16 or VCR, as compared to each agent alone (Table I). In the SK-ES-1 cells, $0.5 \mathrm{mM} \mathrm{NaB}$ and $15 \mathrm{nM}$ Doxo resulted in a 35 and $48 \%$ decrease in cell viability, respectively, but the combination of both resulted in a $83 \%$ decrease (Fig. 2B). In RD-ES cells, the results were similar; $0.75 \mathrm{mM} \mathrm{NaB}$ and $30 \mathrm{nM}$ Doxo resulted in a 33 and $49 \%$ decrease in cell viability, respectively, but the combination of both resulted in a $90 \%$ decrease (Fig. 2B). NaB $(0.5 \mathrm{mM})$ and $0.06 \mu \mathrm{M}$ VP-16 led to a 24 and $30 \%$ decrease in the cell viability of SK-ES-1 cells, respectively, but the combination of both resulted in a $78 \%$ decrease (Fig. 2C). In the RD-ES cells, the results were similar; $0.75 \mathrm{mM} \mathrm{NaB}$ and $0.08 \mu \mathrm{M}$ VP-16 resulted in an 18 and $19 \%$ decrease in cell viability, respectively, but the combination of both resulted in a $67 \%$ decrease (Fig. 2C). Synergistic toxicity was observed in the SK-ES-1 cells when NaB and VCR were combined, however in RD-ES cells the effect of this combination was classified as additive. In the SK-ES-1 cells, $0.5 \mathrm{mM} \mathrm{NaB}$ and $0.5 \mathrm{nM}$ VCR resulted in a 19 and $21 \%$ decrease in cell viability, respectively, but the combination of both resulted in a $61 \%$ decrease (Fig. 2D). In the RD-ES cells, $0.75 \mathrm{mM}$ $\mathrm{NaB}$ and $1.5 \mathrm{nM}$ VCR led to a 33 and $40 \%$ decrease in cell viability, respectively, but the combination of both resulted in a $60 \%$ decrease (Fig. 2D).

Zoledronic acid and traditional antineoplastic agents show synergistic or additive effects on growth inhibition in the ES cell lines. Results showed synergistic toxicity or additive effect in human Ewing sarcoma SK-ES-1 and RD-ES cells at $72 \mathrm{~h}$ when ZA was combined with Doxo, VP-16 or VCR, as compared to each agent alone (Table I). Synergistic toxicity was observed in the SK-ES-1 cells when ZA and Doxo were combined, however in the RD-ES cells the effect of this combination was classified as additive. In the SK-ES-1 cells, $25 \mu \mathrm{M}$ ZA and $15 \mathrm{nM}$ Doxo led to a 46 and $48 \%$ decrease in cell viability, respectively, but the combination of both resulted in a $73 \%$ decrease (Fig. 2E). In the RD-ES cells, $50 \mu \mathrm{M}$ ZA and $30 \mathrm{nM}$ Doxo resulted in a 36 and $47 \%$ decrease in cell viability, respectively, but the combination of both resulted in a $60 \%$ decrease (Fig. 2E). ZA $(25 \mu \mathrm{M})$ and $0.06 \mu \mathrm{M}$ VP-16 resulted in a 44 and a $30 \%$ decrease in the cell viability of SK-ES-1 cells, respectively, but the combination of both resulted in a $70 \%$ decrease (Fig. $2 \mathrm{~F}$ ). In the RD-ES cells, the results were similar: $50 \mu \mathrm{M}$ ZA and $0.08 \mu \mathrm{M}$ VP-16 resulted in a 36 and $19 \%$ decrease in cell viability, respectively, but the combination of both resulted in a $66 \%$ decrease (Fig. 2F). In the SK-ES-1 cells, $25 \mu \mathrm{M}$ ZA and $0.5 \mathrm{nM}$ VCR resulted in a 45 and $25 \%$ decrease in cell viability, respectively, but the combination of both resulted in a $64 \%$ decrease (Fig. 2G). In the RD-ES cells, $50 \mu \mathrm{M}$ ZA and $1.5 \mathrm{nM}$ VCR resulted in a 35 and $41 \%$ decrease in cell viability, respectively, but the combination of both resulted in a $68 \%$ decrease (Fig. 2G). 
A
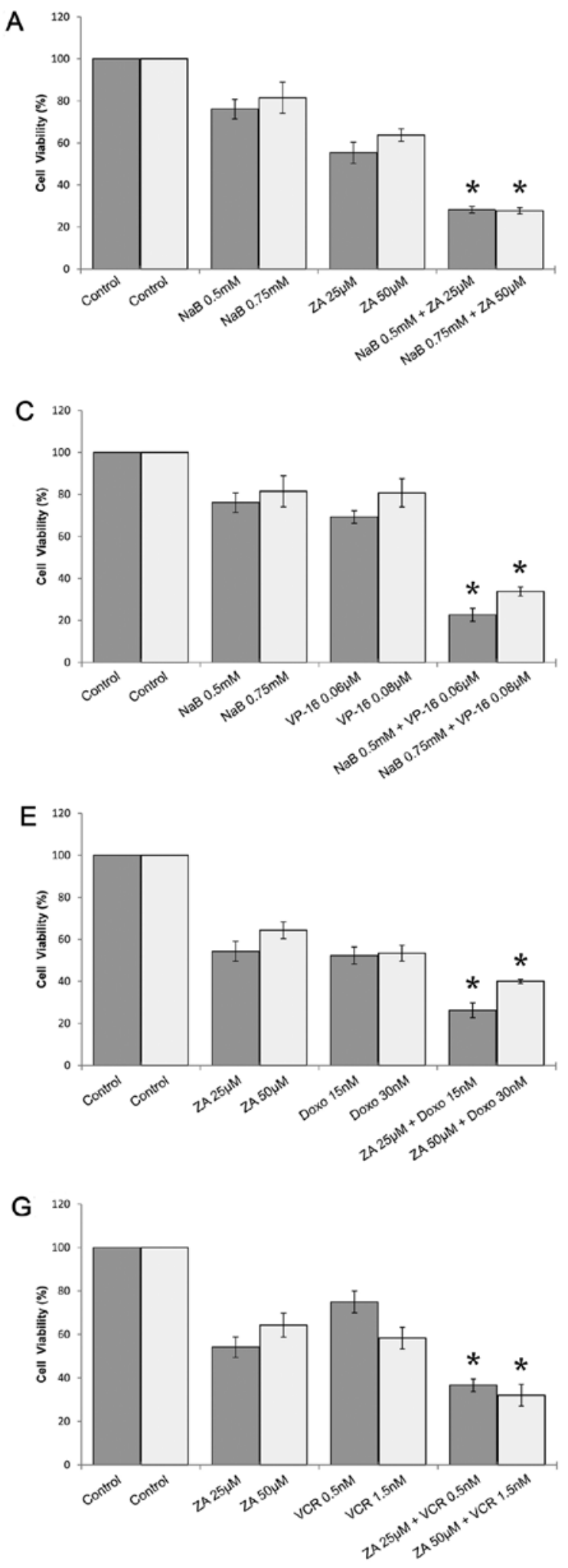
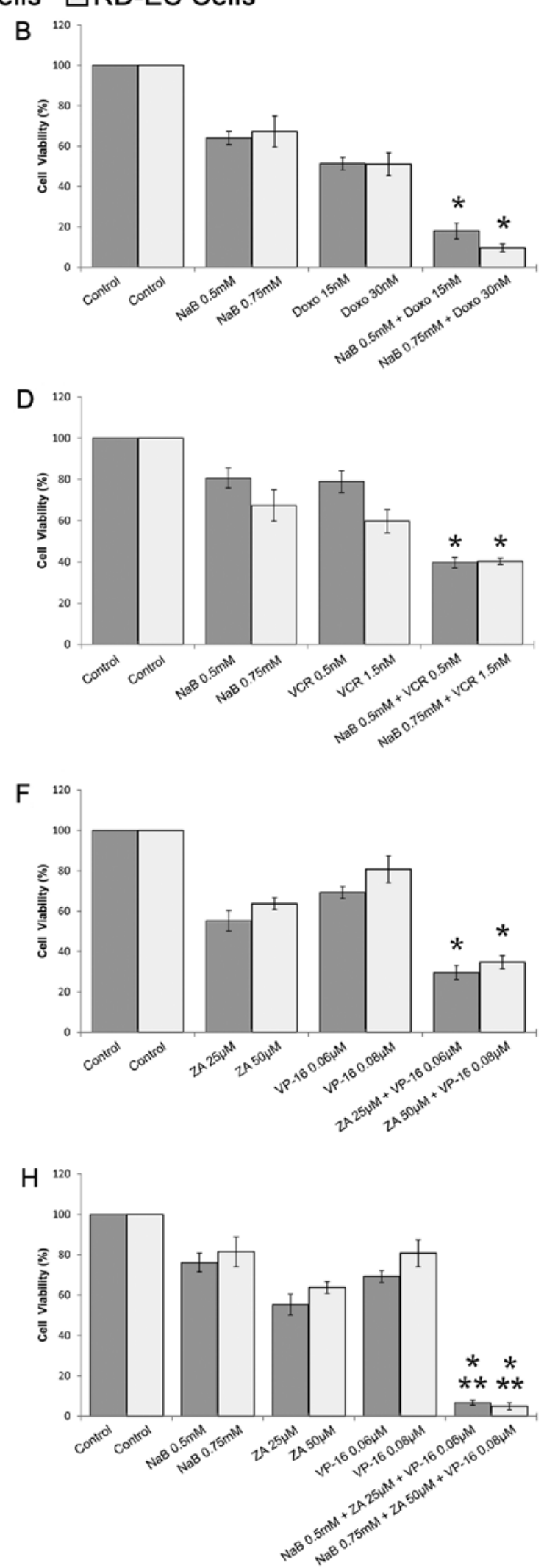

Figure 2. Cytotoxic effects of different combinations of sodium butyrate (NaB), zoledronic acid (ZA), doxorubicin (Doxo), etoposide (VP-16) and vincristine (VCR) on cellular proliferation and viability. SK-ES-1 and RD-ES cell lines were treated for $72 \mathrm{~h}$ with (A) NaB $(0.5$ or $0.75 \mathrm{mM})$ and ZA (25 or $50 \mu \mathrm{M})$, (B) $\mathrm{NaB}(0.5$ or $0.75 \mathrm{mM})$ and Doxo $(15$ or $30 \mathrm{nM}),(\mathrm{C}) \mathrm{NaB}(0.5$ or $0.75 \mathrm{mM})$ and VP-16 $(0.06$ or $0.08 \mu \mathrm{M}),(\mathrm{D}) \mathrm{NaB}(0.5$ or $0.75 \mathrm{mM})$ and $\mathrm{VCR}(0.5 \mathrm{or} 1.5 \mathrm{nM})$, (E) ZA $(25$ or $50 \mu \mathrm{M})$ and Doxo $(15$ or $30 \mathrm{nM})$, (F) ZA $(25$ or $50 \mu \mathrm{M})$ and VP-16 $(0.06$ or $0.08 \mu \mathrm{M})$, (G) ZA $(25$ or $50 \mu \mathrm{M})$ and VCR $(0.5 \mathrm{or} 1.5 \mathrm{nM}),(\mathrm{H}) \mathrm{NaB}$ $(0.5$ or $0.75 \mathrm{mM})$, VP-16 $(0.06$ or $0.08 \mu \mathrm{M})$ and ZA $(25$ or $50 \mu \mathrm{M})$, respectively. Cellular proliferation and viability were assessed in triplicates, in at least three independent experiments. Data are expressed as mean percentage of the controls, and standard deviations (SD) are shown in the error bars. P-values (P) were determined using a one-way analysis of variance (ANOVA), followed by the Tukey-Kramer test, with $\mathrm{P}<0.05$ considered to indicate statistically significant differences. All treatments led to a statistically significant reduction in cell growth when compared to the controls. ${ }^{*} \mathrm{P}<0.05$ vs. single agents; ${ }^{* * *} \mathrm{P}<0.05$ vs. combined treatments. 
A
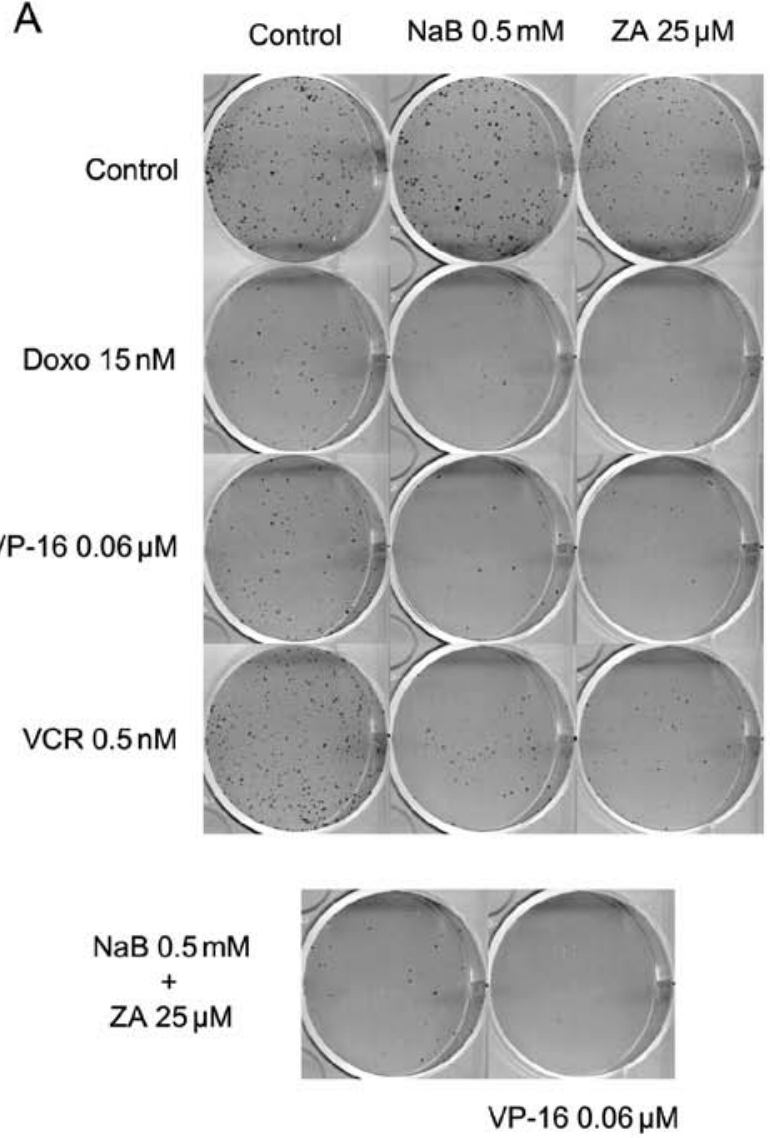

B

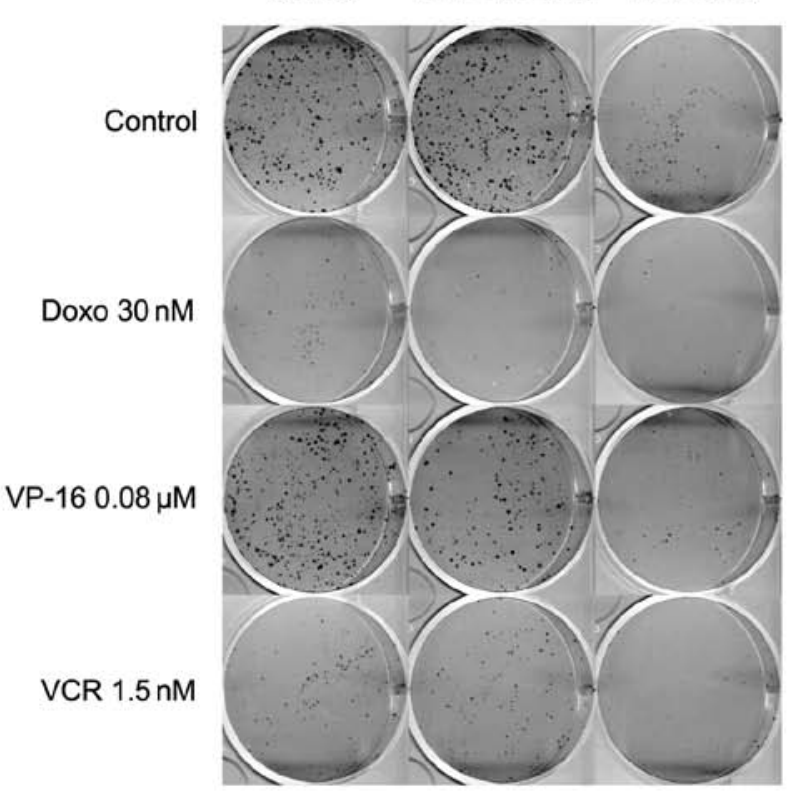

\author{
$\mathrm{NaB} 0.75 \mathrm{mM}$ \\ $+$ \\ ZA $50 \mu \mathrm{M}$
}

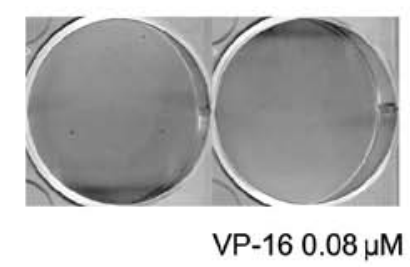

Figure 3. Representative images of cell survival experiments performed in triplicate with (A) SK-ES-1 and (B) RD-ES cells.

Strong synergistic growth inhibition by sodium butyrate, zoledronic acid and etoposide in the ES cell lines. A synergistic growth inhibition effect was observed when combining etoposide with sodium butyrate or etoposide with zoledronic acid as compared to each agent alone, in both SK-ES-1 and RD-ES Ewing sarcoma cancer cells. Therefore, it was postulated that this effect might increase by combining the three agents (triple treatment). Results showed a strong synergistic toxicity in SK-ES-1 and RD-ES cells at $72 \mathrm{~h}$, as compared to each agent alone (Table I). The triple treatment $(0.5 \mathrm{mM}$ $\mathrm{NaB}$ plus $25 \mu \mathrm{M}$ ZA plus $0.06 \mu \mathrm{M}$ VP-16) resulted in a $94 \%$ decrease in cell viability of SK-ES-1 cells. This reduction was higher than that observed in the double treatments $(\mathrm{NaB}$ plus ZA, 72\%; NaB plus VP-16, 78\% and ZA plus VP-16, 70\%) and much greater than that observed when each agent was administered alone (NaB, 24\%; ZA, 44\% and VP-16, 31\%) (Fig. 2H). In RD-ES cells, the results were similar; the $0.75 \mathrm{mM} \mathrm{NaB}$ plus $50 \mu \mathrm{M}$ ZA plus $0.08 \mu \mathrm{M}$ VP-16 combination resulted in a $96 \%$ decrease in cell viability. This reduction was higher than those obtained with the double treatments $(\mathrm{NaB}$ plus $\mathrm{ZA}$, $3 \%, \mathrm{NaB}$ plus VP-16, $67 \%$ or ZA plus VP-16, 66\%) and much higher than those obtained with each agent alone $(\mathrm{NaB}, 18 \%$, ZA, 36\%, or VP-16, 19\%) (Fig. 2H).

Combination of sodium butyrate and zoledronic acid significantly suppresses colony formation in the ES cell lines. To analyze the effects of $\mathrm{NaB}$ and $\mathrm{ZA}$ on the survival and proliferation of SK-ES-1 and RD-ES cells, a clonogenic assay was performed. As shown in Figs. 3A and 4A, NaB did not significantly suppress colony formation of SK-ES-1 cells (6\%), while ZA suppressed colony formation by $62 \%$. Co-treatment with $\mathrm{NaB}$ and ZA though, strongly suppressed colony formation $(84 \%)$ at the doses tested. In RD-ES cells, the results were similar; NaB did not reduce colony formation $(102 \%$, not significant), while ZA suppressed colony formation by $63 \%$ and the $\mathrm{NaB}$ plus $\mathrm{ZA}$ co-treatment strongly suppressed colony formation (85\%) (Figs. 3B and 4A).

Co-treatment with sodium butyrate and traditional antineoplastic agents significantly reduces colony formation in the ES cell lines. In the SK-ES-1 cells, at the doses tested, $\mathrm{NaB}$ did not reduce colony formation, whereas Doxo reduced it by $68 \%$. The combination of both strongly suppressed colony formation (92\%) (Figs. 3A and 4B). In the RD-ES cells, the results were similar; $\mathrm{NaB}$ did not reduce colony formation, (4\%, not significant), Doxo suppressed it by $64 \%$, and co-treatment with both agents strongly suppressed colony formation (92\%) (Figs. 3B and 4B). The combination of NaB and VP-16, at the doses tested, strongly suppressed colony formation in SK-ES-1 cells (86\%) and suppressed colony formation in RD-ES cells (64\%). NaB alone did not significantly reduce colony formation in both cell lines, and VP-16 alone reduced colony formation in SK-ES-1 and RD-ES cells by 45 and 24\%, respectively (Figs. 3A and B and $4 \mathrm{C}$ ). At the doses tested, the combination of $\mathrm{NaB}$ and VCR suppressed colony formation by $44 \%$. When compared to the single treatments with $\mathrm{NaB}$ 


\section{$\square$ SK-ES-1 Cells $\square$ RD-ES Cells}
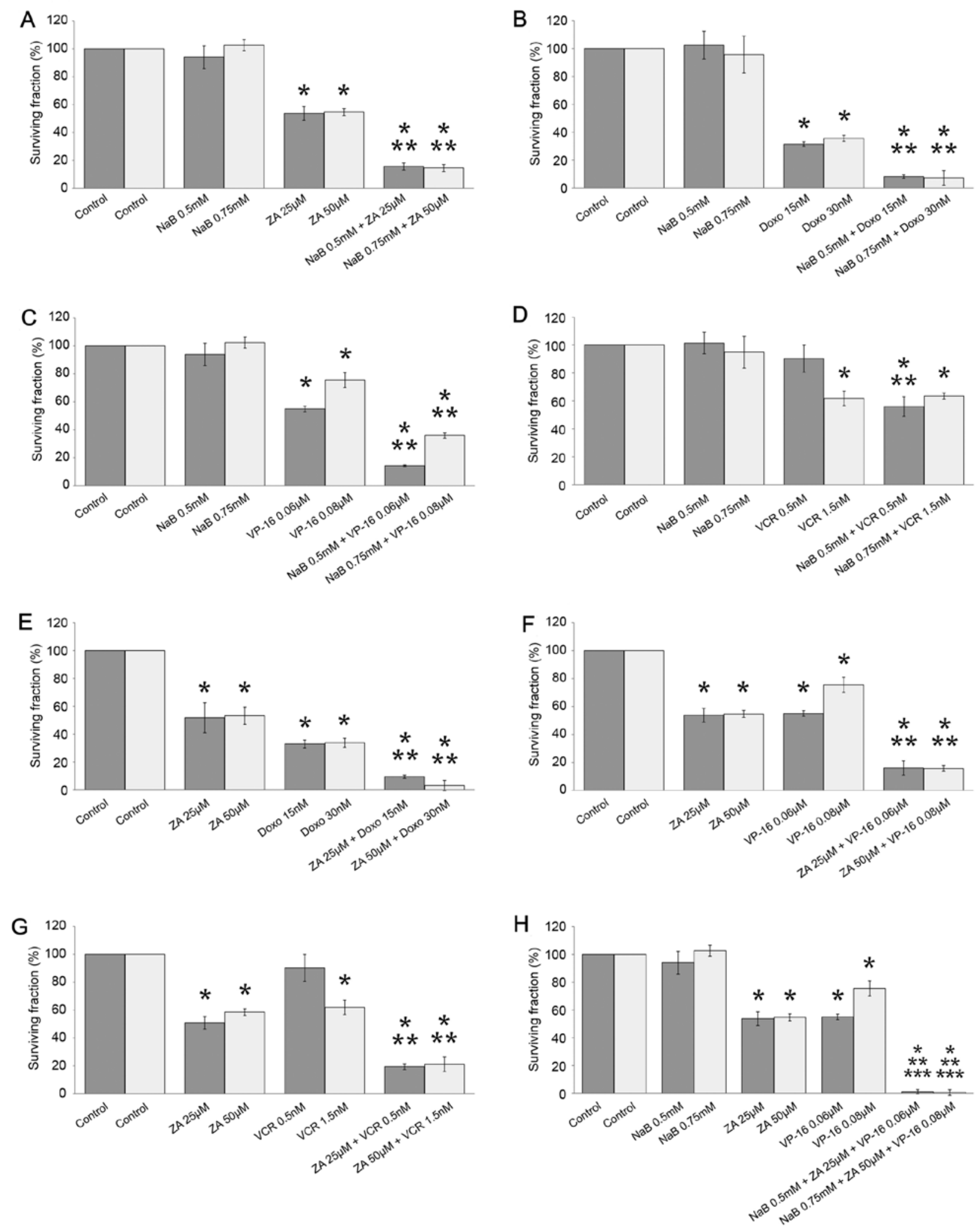

Figure 4. Effects of different combinations of sodium butyrate (NaB), zoledronic acid (ZA), doxorubicin (Doxo), etoposide (VP-16) and vincristine (VCR) on suppression of colony formation in Ewing sarcoma (ES) cells. Colony formation of SK-ES-1 and RD-ES cells treated with (A) NaB (0.5 or $0.75 \mathrm{mM})$ and ZA $(25$ or $50 \mu \mathrm{M})$, (B) $\mathrm{NaB}(0.5$ or $0.75 \mathrm{mM})$ and Doxo $(15$ or $30 \mathrm{nM}),(\mathrm{C}) \mathrm{NaB}(0.5$ or $0.75 \mathrm{mM})$ and VP-16 $(0.06$ or $0.08 \mu \mathrm{M}),(\mathrm{D}) \mathrm{NaB}(0.5$ or $0.75 \mathrm{mM})$ and VCR $(0.5$ or $1.5 \mathrm{nM})$, (E) ZA (25 or $50 \mu \mathrm{M})$ and Doxo (15 or $30 \mathrm{nM})$, (F) ZA (25 or $50 \mu \mathrm{M})$ and VP-16 $(0.06$ or $0.08 \mu \mathrm{M}),(\mathrm{G}) \mathrm{ZA}(25$ or $50 \mu \mathrm{M})$ and VCR $(0.5$ or $1.5 \mathrm{nM}),(\mathrm{H}) \mathrm{NaB}(0.5$ or $0.75 \mathrm{mM})$, VP-16 $(0.06$ or $0.08 \mu \mathrm{M})$ and ZA $(25$ or $50 \mu \mathrm{M})$, respectively, for $72 \mathrm{~h}$. The assay was initiated by plating $1 \times 10^{3}$ cells/well into 6-well plates. After 10-14 days, cells were fixed and stained with crystal violet as described in Material and methods. The formation of colonies from the clonogenic assay was analyzed by optimized digital counting using ImageJ software. The results are shown as the mean percentage of the surviving fraction, and standard deviations (SD) are shown in the error bars. P-values (P) were determined using a one-way analysis of variance (ANOVA), followed by the Tukey-Kramer test, with $\mathrm{P}<0.05$ considered to indicate statistically significant differences. ${ }^{*} \mathrm{P}<0.05$ vs. control; ${ }^{* *} \mathrm{P}<0.05$ vs. single agents; ${ }^{* * * *} \mathrm{P}<0.05$ vs. combination treatments. 

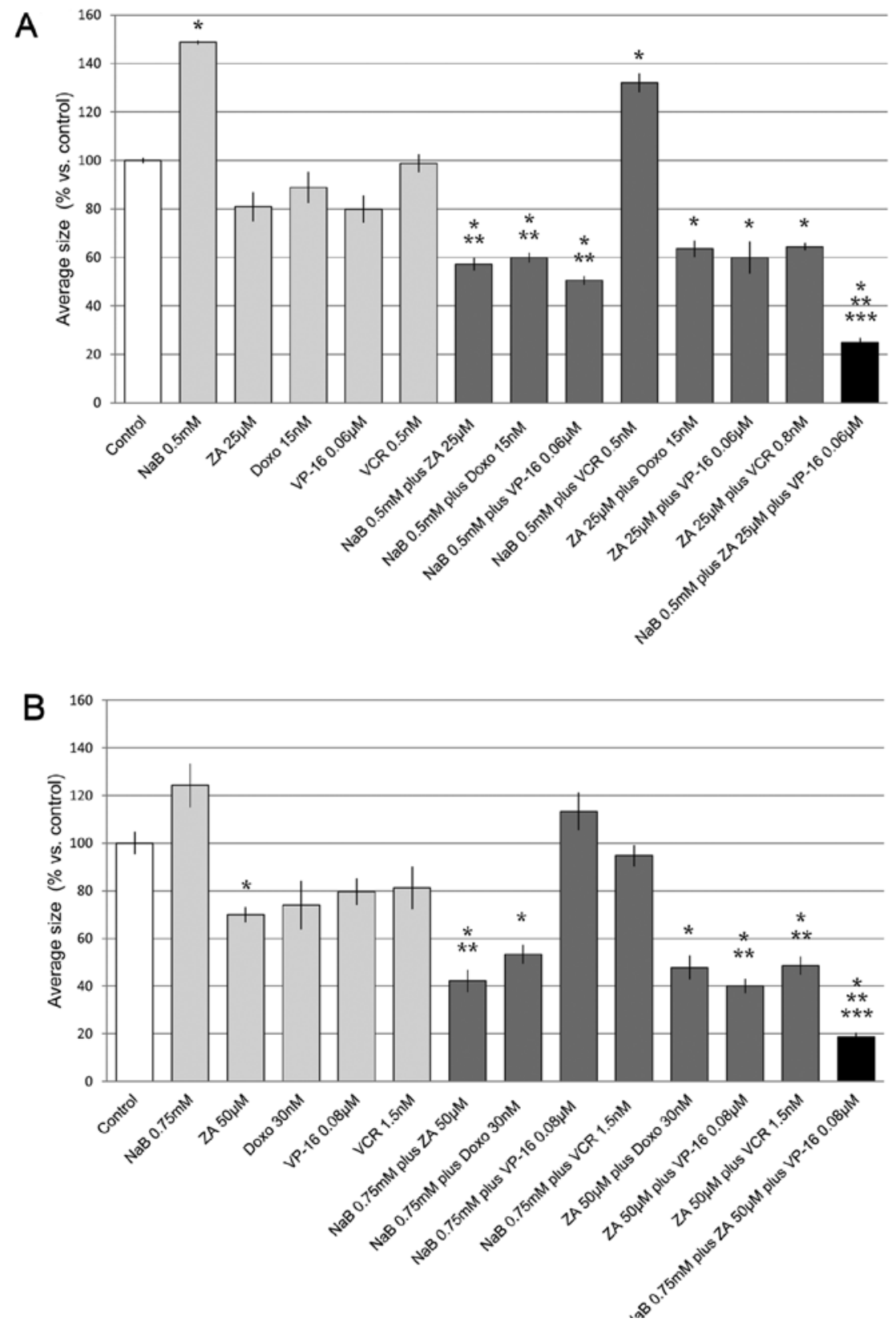

Figure 5. Effects of sodium butyrate ( $\mathrm{NaB}$ ), zoledronic acid (ZA), doxorubicin (Doxo), etoposide (VP-16), vincristine (VCR) and different combinations of these drugs on the average size of the colonies formed in the clonogenic assay in Ewing sarcoma (ES) cells. Following estimation of the number of colonies formed in the clonogenic assay, their average size was determined by optimized digital counting using Image J software in SK-ES-1 (A) and RD-ES (B) cells. The average size of the colonies for each treatment is expressed as mean percentage of the controls, and standard deviations (SD) are shown in the error bars. $\mathrm{P}$-values $(\mathrm{P})$ were determined using a one-way analysis of variance (ANOVA), followed by the Tukey-Kramer test, with $\mathrm{P}<0.05$ considered to indicate statistically significant differences. ${ }^{*} \mathrm{P}<0.05$ vs. control; ${ }^{* *} \mathrm{P}<0.05$ vs. single agents; ${ }^{* * *} \mathrm{P}<0.05$ vs. combination treatments.

or VCR, colony formation was not significantly reduced in SK-ES-1 cells (Figs. 3A and 4D). However, in the RD-ES cells, co-treatment with $\mathrm{NaB}$ and VCR did not significantly reduce colony formation when compared to VCR alone (38 and 36\%, respectively) (Figs. 3B and 4D).

Zoledronic acid, traditional antineoplasic agents and sodium butyrate significantly suppress colony formation in the ES cell lines. In the SK-ES-1 cells, at the doses tested, ZA and Doxo mono-treatment suppressed colony formation by 48 and $67 \%$, respectively. The combination of both strongly suppressed colony formation (91\%) (Figs. 3A and 4E). In the RD-ES cells, the results were similar; ZA and Doxo mono-treatment suppressed colony formation by 46 and 66\%, respectively, and the combination of both drastically suppressed colony formation (97\%) (Figs. 3B and 4E). ZA and VP-16 mono-treatment suppressed colony formation by 47 and $45 \%$, respectively. The combination of both strongly suppressed colony formation (84\%) at the doses tested in the SK-ES-1 cells (Figs. 3A and $4 \mathrm{~F}$ ). In the RD-ES cells, the results were similar; ZA suppressed colony formation by $45 \%$, VP-16 weakly suppressed colony formation (24\%) and the combination of both strongly suppressed colony formation (83\%) (Figs. 3B and $4 \mathrm{~F}$ ). At the doses tested, ZA suppressed colony formation 
by $49 \%$, whereas VCR did not significantly reduce colony formation (10\%). However, combined treatment with ZA and VCR strongly suppressed colony formation (81\%) (Figs. 3A and $4 \mathrm{G})$. In the RD-ES cells, the results were similar; ZA or VCR suppressed colony formation (41 and 38\%, respectively) and the combination of both strongly suppressed colony formation (79\%) (Figs. 3B and 4G). The triple treatment combining NaB, ZA and VP-16 at the doses tested, drastically suppressed colony formation in SK-ES-1 (99\%) and in RD-ES $(100 \%)$ cells. These effects were more pronounced than those obtained with treatments combining only two agents $(\mathrm{NaB}$ plus ZA, NaB plus VP-16) and were much greater than those obtained with each agent alone (Figs. 3A and $3 \mathrm{~B}$ and $4 \mathrm{H}$ ).

Effects of sodium butyrate, zoledronic acid, doxorubicin, etoposide, vincristine and combinations of these on the average size of ES colonies. Through the use of optimized digital counting, it was possible to evaluate not only the surviving fraction of the colonies, but also their average size. This is important for a more accurate assessment of the longterm effects of the tested compounds. In SK-ES-1 cells, NaB significantly enhanced the average colony size (48\%), while ZA, Doxo, VP-16 and VCR did not alter the average size of the colonies formed in the clonogenic assay as compared to control $(20,12,20$ and 1\%, respectively; Fig. 5A). The combined treatments with $\mathrm{NaB}$ plus $\mathrm{ZA}, \mathrm{NaB}$ plus Doxo, $\mathrm{NaB}$ plus VP-16, ZA plus Doxo, ZA plus VP-16, ZA plus VCR and NaB plus ZA plus VP-16 reduced colony size (43, $41,50,37,40,36$ and $76 \%$, respectively), whereas the $\mathrm{NaB}$ plus VCR co-treatment increased (32\%) the average size of the colonies as compared to the control (Fig. 5A). The NaB plus ZA, NaB plus Doxo and NaB plus VP-16 combinations significantly reduced the average colony size as compared to each drug alone (Fig. 5A). The NaB plus VCR combination increased the average size of the colonies as compared to VCR alone and this effect was statistically similar to that obtained with the NaB mono-treatment (Fig. 5A). A slight reduction was observed when ZA plus Doxo, ZA plus VP-16, and ZA plus VCR combinations were compared to each drug alone, however these reductions were not significant (Fig. 5A). The combination of $\mathrm{NaB}, \mathrm{ZA}$ and VP-16 strongly reduced the average size of the colonies. This reduction was higher that the one obtained with the double treatments $(\mathrm{NaB}$ plus $\mathrm{ZA}$, $\mathrm{NaB}$ plus VP-16 and ZA plus VP-16) and much greater than those obtained with each agent alone (Fig. 5A). As shown in Fig. 5B, NaB enhanced the average size of RD-ES colonies by $24 \%$, while ZA, Doxo, VP-16 and VCR reduced the average size of RD-ES colonies when compared to the control (29, 26, 20 and $19 \%$, respectively). However, the only statistically significant difference was observed when ZA was compared to its control. The combined treatments with $\mathrm{NaB}$ plus ZA, NaB plus Doxo, ZA plus Doxo, ZA plus VP-16, ZA plus VCR and $\mathrm{NaB}$ plus $\mathrm{ZA}$ plus $\mathrm{VP}-16$ reduced colony size $(58,47$, $53,60,52$ and $82 \%$, respectively), whereas NaB plus VP-16 increased (13\%, not significant) and $\mathrm{NaB}$ plus VCR reduced (5\%, not significant) the average size of the colonies when compared to controls (Fig. 5B). The NaB plus ZA, ZA plus VP-16 and ZA plus VCR co-treatments reduced the average size of the colonies when compared to each drug alone (Fig. 5B). Co-treatments with $\mathrm{NaB}$ plus VCR and NaB plus
VP-16 increased colony average size when compared with VCR and VP-16 alone (respectively) and the strength of the observed increase was similar to that obtained with the $\mathrm{NaB}$ mono-treatment (Fig. 5B). A slight reduction in colony size was observed when NaB plus Doxo and ZA plus Doxo co-treatments were compared to each drug alone, however these reductions were not significant (Fig. 5B). As observed in the SK-ES-1 cells, the combination of NaB, ZA and VP-16 strongly reduced the average size of the colonies formed by RD-ES cells. This reduction was more pronounced than the ones obtained with the double treatments ( $\mathrm{NaB}$ plus $\mathrm{ZA}, \mathrm{NaB}$ plus VP-16 and ZA plus VP-16) and much greater than the ones obtained with each agent alone (Fig. 5B).

\section{Discussion}

HDAC inhibitors (HDIs) and bisphosphonates are considered as promising alternatives in the treatment of cancer. Among the different childhood sarcomas, Ewing sarcoma (ES) appears particularly suitable for treatment with zoledronic acid because of its role in the modulation of the bone microenvironment (30).

In ES cells, the effects of HDIs have been evaluated. Sakimura et al (31) demonstrated that the abnormal functions of the EWS-FLI1 fusion oncoprotein are associated with both histone acetylation and deacetylation (EWS-FLI1 represses HAT and activates HDAC), suggesting that HDIs might serve as a molecular-based therapeutic application for ESFTs. Furthermore, another study concluded that HDIs may provide a novel strategy for treatment of ES, either applied as a monotreatment or in combination with TRAIL (8). The molecular mechanisms by which HDIs exert antitumor effects are not well known. In ESFTs, some studies have been undertaken to evaluate the effects of acetylation by the EWS-FLI1 protein $(32,33)$.

Among the different classes of compounds studied for the treatment of ES, the bisphosphonates have been the most intensely evaluated, since ES is characterized by a marked bone resorption. Therefore, any therapeutic strategy that targets osteoclasts, such as the bisphosphonates, is promising $(2,4,34)$. Potential antitumor effects observed in osteosarcoma might be explained by a direct activity on tumor cells (35), by a strong modulation of the tumor microenvironment $(36,37)$, or by stimulation of immune effectors $(38,39)$. In ES, bisphosphonates appear to have similar inhibitory activities $(21,22,40)$. The results obtained by Zhou et al (22) suggest that ZA might represent a novel therapeutic approach for the treatment of patients with ES, since it induces apoptosis and inhibits primary bone tumor growth via a mechanism involving the upregulation of osteoprotegerin. Moreover, ZA showed a synergistic effect with paclitaxel in the TC71 human ES cell line, both in vitro and in animal models (22). Other studies have concluded that ZA is a new adjuvant therapeutic strategy allows a decrease in the dosage of chemotherapeutic agents employed for treating ES patients, since ZA, when combined with ifosfamide, exhibited synergistic effects in a soft tissue model: its combination with one cycle of ifosfamide resulted in an inhibitory effect comparable to three cycles of ifosfamide alone (21). In the clinic, one case was reported where ZA combined with chemotherapy was an effective 
salvage treatment for recurrent ES (41). The use of zoledronic acid in combination with first-line chemotherapy for localized ES is being addressed in Europe, in randomized phase III trials (Ewing 2008 and Euro-Ewing 2012). In juvenile models, zoledronic acid decreases endochondral bone growth in a reversible manner $(30,34)$.

Recent studies strongly suggest that successful cancer therapy is likely to involve a combination of HDIs or bisphosphonates with each other or with traditional chemotherapeutic agents (13-22). Although the associations between HDIs or bisphosphonates and traditional anticancer drugs $(18,42,43)$ and between HDIs and ZA (44) have been evaluated in other types of cancer, to our knowledge, this is the first study to evaluate the effects of these combinations in ES.

In the present study, the experiments were carefully designed to evaluate how the co-treatment with antineoplastic drugs and $\mathrm{NaB}$ or ZA, and with $\mathrm{NaB}$ and ZA, may affect in vitro cellular proliferation. Dose curves were calculated so as to establish the lowest concentrations that significantly reduced cellular proliferation when compared to the controls, albeit without causing significant cell death. Administering these drugs at lower concentrations allowed us to assess with greater certaintly whether $\mathrm{NaB}$ and/or ZA enhance the effects elicited by each drug when administered in combination. We hypothesized that $\mathrm{NaB}$ and $\mathrm{ZA}$ might show synergistic cytotoxic effects when combined or in association with traditional antineoplastic drugs, in the human ES cell lines SK-ES-1 and RD-ES. Our results clearly demonstrated that HDIs, NaB and ZA, when employed alone, significantly inhibited proliferation and viability of both cell lines. More importantly, the combination of $\mathrm{NaB}$ with $\mathrm{ZA}$ exhibited a synergistic cytotoxic effect on both ES cell lines as compared to each agent alone. Furthermore, these results were confirmed by clonogenic assays, suggesting that the effects were extended to, or intensified, for a longer period of time. The co-treatments with $\mathrm{NaB}$ or $\mathrm{ZA}$ and traditional chemotherapy agents also showed acute $(72 \mathrm{~h})$ and chronic (10-14 days) synergistic cytotoxic effects in SK-ES-1 cells as compared to each agent alone. As clearly demonstrated by the results, the RD-ES cell line was more resistant to these treatments. The combined treatments with $\mathrm{NaB}$ plus VCR and ZA plus Doxo, for example, demonstrated only an additive effect in RD-ES cells when compared to each agent alone at $72 \mathrm{~h}$. The effect of ZA and Doxo had long-term intensity (10-14 days), while this result was not observed for the combination of $\mathrm{NaB}$ with VCR. On the other hand, the chemotherapeutic agent etoposide (VP-16), in combination with $\mathrm{NaB}$ or ZA, demonstrated a synergistic cytotoxic effect, and in combination with $\mathrm{NaB}$ and $\mathrm{ZA}$ (triple treatment) showed a strong synergistic cytotoxic effect in both ES cell lines as compared to any agent alone. These results were also confirmed by colony formation assays. An additional evaluation of importance was the determination of the average colony size in the clonogenic assay. This measure showed that $\mathrm{NaB}$ alone or in any combination promoted an increase in the average size of the colonies of both cell lines when compared to the controls. On the other hand, ZA alone or in combination with different agents not only reduced the number of colonies, but also reduced the average colony size in both cell lines. Overall, these results suggest that the combined treatments with $\mathrm{NaB}$ plus ZA, $\mathrm{NaB}$ or ZA plus Doxo and particularly, $\mathrm{NaB}$ and/or ZA with VP-16 represent the most promising alternatives for the development of new therapies for Ewing sarcoma.

The use of HDIs, bisphosphonates and traditional antineoplastic drugs as monotherapeutic agents has some limitations. Recent findings suggest that clinical use of HDAC inhibitors for conditions such as cancer and a multitude of other ailments might have undesirable effects on bone cell populations $(45,46)$. Moreover, Battaglia et al suggested that endochondral bone growth is transiently disturbed by high doses of ZA, administered during the pediatric treatment of primary bone tumors (34). It is unclear whether concurrent administration of an osteogenic therapy (e.g., bisphosphonates) can attenuate HDI-induced bone loss (46). On the other hand, the negative skeletal consequences of broad-acting HDIs, currently under clinical trials, might be considered as acceptable side-effects from oncologists for patients with sarcoma or advanced metastatic tumors, due to the beneficial effects of HDIs on tumors within the bone (45). It is well established that the traditional chemotherapeutic agents tested in the present study cause a number of undesirable side-effects in patients. Furthermore, while undergoing treatment, several patients become irresponsive to these agents, especially when the latter are employed as monotherapy. Since the $\mathrm{NaB}$ plus ZA combination demonstrated a synergistic cytotoxic effect, and $\mathrm{NaB}$ or ZA can potentiate the effects of traditional cytotoxic drugs, these co-treatments might allow such drugs to be administered in smaller doses, while preserving their effects. This fact consolidates the idea that using $\mathrm{NaB}$ and $\mathrm{ZA}$ as anticancer adjuvant drugs might decrease the toxic side-effects that are common to the current anticancer protocols. This idea is further supported by clinical studies that have shown that histone hyperacetylation can be safely achieved in humans, and that treatment with these agents is plausible (13-15). The clinical use of these co-treatments could reduce frequent disease complications and allow to bypass the need for dose reductions or temporary discontinuations of treatment as a means to address toxicity or drug resistance. This approach has the potential to improve the chances of success and the quality of life of patients per se. The results from the present study provide a basis for evaluating, in clinical trials, the combinations tested herein, as well as the effects of administering traditional anticancer drugs combined with $\mathrm{NaB}$ and/or ZA in lower concentrations.

An additional mechanism that remains largely unknown is the one by which the combination of bisphosphonates, HDIs and traditional chemotherapeutic agents exerts synergistic antitumor effects. As described in the literature, traditional drugs, such as doxorubicin and etoposide (VP-16), cause DNA damage (Doxo by DNA intercalation, macromolecular biosynthesis and topoisomerase II inhibition and VP-16 by topoisomerase II inhibition). Vincristine inhibits microtubule dynamics, which results in mitotic arrest and eventually, cell death. These anticancer drugs can inhibit cellular proliferation via p53-dependent and p53-independent mechanisms, resulting in cell cycle arrest and/or apoptosis. In vitro studies have shown clear antitumor effects for BPs, particularly zoledronic acid, as demonstrated by the BP-mediated induction of tumor cell apoptosis and their inhibitory effects on tumor cell adhesion, invasion, tumor cell viability, proliferation and angiogenesis (22). The mechanisms underlying this spectrum of antitumor 


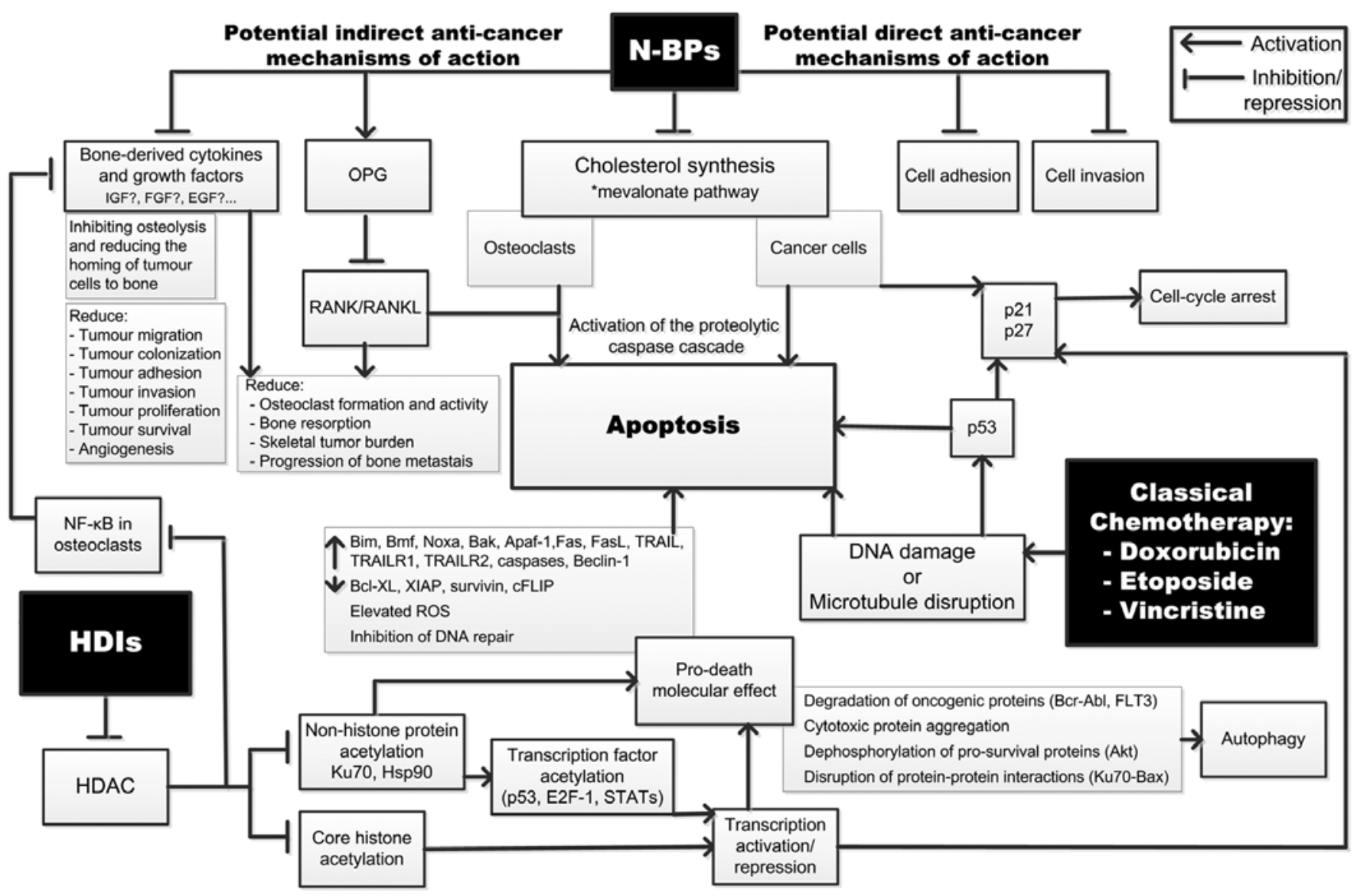

Figure 6. Schematic representation of the antitumor activity of the nitrogen-containing bisphosphonate family (N-BP), histone deacetylase inhibitors (HDIs) and traditional chemotherapeutic drugs (doxorubicin, etoposide and vincristine) as shown in pre-clinical studies. Recent studies have demonstrated synergistic antitumor effects when combining BPs, HDIs and cytotoxic agents. The molecular mechanisms by which these combinations exert their antitumor effects have not yet been elucidated, but it is possible that they constitute a complex combination of induction of proapoptotic proteins and suppression of cell cycle proteins.

activities are still under investigation. It was proposed that BPs exert both indirect and direct antitumor cell effects by inhibiting the farnesyl diphosphonate synthase in the mevalonate pathway. Cholesterol synthesis via the mevalonate pathway is essential for all nucleated cells and, therefore, any cell type that metabolizes N-BPs may be affected, including tumor cells (direct antitumor effects) and osteoclasts (indirect antitumor effects). Zoledronic acid-induced tumor cell apoptosis was demonstrated to be associated with the release of cytochrome $C$ and the resulting activation of the caspase pathway (47). This might occur via the inhibition of the mevalonate pathway and the consequent inhibition of prenylation of essential signaling $\mathrm{G}$ proteins such as Ras, Rac and Rho. As inhibitors of bone resorption, BPs reduce the release of bone-derived cytokines and growth factors, essentially rendering bone tissue less attractive to tumor cells as a site for tumor migration, colonization, adhesion and invasion, proliferation and survival. Furthermore, especially in bone tumors, BPs might also exert additional indirect antitumor effects of importance, since these molecules inhibit RANK expression and osteoclast progenitor migration during osteoclastogenesis while they increase osteoprotegerin (OPG) expression $(22,30,47-49)$. HDAC inhibitors (HDIs) are enzymes that modify chromatin access to transcription factors and to gene transcription. HDIs are potent inducers of tumor cell apoptosis, which can be mediated by regulating histone function and subsequently, gene transcription, and/or by regulating the function or stability of non-histone proteins. While induction of apoptosis appears to be the predominant mechanism of HDI-mediated cell death, alternative cell death mechanisms, such as autophagy, might be stimulated when apoptotic proteins or pathways are disabled. However, the molecular events required for HDI-mediated autophagy are poorly understood. Depending on the stimulus, apoptosis can proceed via two functionally distinct pathways, the 'extrinsic' death receptor pathway and the 'intrinsic' mitochondrial pathway. Although the initiating phases of the two pathways are molecularly separate, a number of the downstream events, such as the activation of caspase-3, are shared between the two pathways and, therefore, the biochemical and morphological features of intrinsic and extrinsic apoptosis (including phosphatidylserine exposure, effector caspase activation and DNA fragmentation) are similar. HDIs may sensitize tumor cells to DNA-damaging agents through one or more of the following mechanisms. First, according to a relatively simplistic view, reversal of chromatin condensation following HDI-mediated hyperacetylation of core histones might increase the accessibility of DNA-damaging agents to their targets and thus, enhance their cytotoxic effects. Second, chromatin remodeling by HDIs can induce a DNA damage-like response that involves the activation of the DNA damage kinase ATM, and 
can enable the formation of DNA strand breaks. In ES cells, EWS-FLI1 represses HAT and activates HDAC. HDAC inhibition restores HAT activity, inhibits cell growth and induces apoptosis. The HDI FK228 decreases EWS-FLI1 expression and ES proliferation and induces TRAIL-dependent apoptosis. Furthermore, HDIs inhibit tumor proliferation and/or survival and the associated inflammation/bone resorption via neutralization of NF- $\kappa \mathrm{B}$ in osteoclasts. This dual effect of HDIs could lead to reduced levels of proinflammatory cytokines and growth factors in the tumor microenvironment and, thus, contribute to tumor regression (30,50-52).

We present a schematic representation of the main established anticancer mechanisms involving BPs, HDIs and traditional chemotherapeutic drugs in Fig. 6. Based on this overview scheme, one might infer that the molecular mechanisms by which the co-treatment with $\mathrm{NaB}, \mathrm{ZA}$ and the traditional drugs tested in the present study exert their effects are a complex combination of induction of proapoptotic proteins and suppression of cell cycle proteins. This hypothesis is supported by studies that evaluated similar associations in ES or other types of cancer. For instance, Zhou et al (22), using a primary ES animal model in nude mice, demonstrated that zoledronic acid significantly inhibits osteolytic ES growth in vivo, enhances tumor sensitivity to paclitaxel and exhibits a synergistic apoptotic effect when combined with paclitaxel. Ottewell et al (49) studied the anticancer effects of doxorubicin and zoledronic acid on the metastatic growth of breast cancer tumors in the bone. They showed that the in vivo administration of doxorubicin followed by zoledronic acid caused specific changes in gene/protein expression and inhibited the mevalonate pathway in breast tumors metastasizing in the bone. The roles of doxorubicin and zoledronic acid in induction of apoptosis of tumor cells growing in the bone are unclear. Both drugs have been shown to induce apoptosis by disrupting the balance of proteins of the $\mathrm{Bcl}-2$ family and mitochondrial membrane potential, leading to an activation of caspase- 9 and to cleavage of caspase- 3 . In addition to activating the mitochondrial pathway, both drugs can induce the cleavage of caspase- 8 , resulting in activation of the mitochondrial-independent pathway. According to the authors, the induction of apoptosis following sequential treatment with doxorubicin and zoledronic acid could be also caused by one drug increasing the cytotoxicity of the other, or could be the result of an additive effect of both drugs acting on the same pathway (49).

Regarding the associations between HDIs and BPs, Sonnemann et al found that the suberoylanilide hydroxamic acid (SAHA) and ZA interacted cooperatively to induce cell death in prostate carcinoma cells, and that this combination was clearly synergistic in LNCaP and PC-3 cell lines (44). Furthermore, experiments with two other HDIs, sodium butyrate and MS-275, revealed that the effect of this combination was not specific to SAHA. The coadministration of SAHA and ZA resulted in the initiation of apoptosis, potently activated caspase-3 and led to the accumulation of sub-G1 cells. SAHA and ZA synergistically affected mitochondrial function, indicating that the activities of these agents converge upstream of the mitochondria. In addition, experiments using the polycaspase inhibitor $\mathrm{z}-\mathrm{VAD}$-fmk revealed that caspase activation was required for the cytotoxic effects observed when combining SAHA and ZA, as measured by propidium iodide uptake and by quantifying DNA fragmentation, further substantiating that the SAHA/ZOL-induced cell death is due to apoptosis. Since ZA acts by inhibiting the mevalonate pathway thereby preventing protein prenylation, the authors explored whether the mevalonate pathway is also the target of the cooperative action of ZA and SAHA. They found that geranylgeraniol, but not farnesol, significantly reduced ZA/SAHA-induced cell death, indicating that the synergistic action of the agents was due to the inhibition of geranylgeranylation. Consistent with this hypothesis, a direct inhibitor of geranylgeranylation, GGTI-298, was found to synergize with SAHA to induce cell death, whereas an inhibitor of farnesylation, FTI-277, had no effect. In addition, SAHA synergized with mevastatin, an inhibitor of the proximal enzyme in the mevalonate pathway. Therefore, we argue that these are the key pathways for a better understanding of the mechanisms by which the tested combinations exert synergistic anticancer effects. Our future efforts will focus on the better understanding of these mechanisms.

The study presented here is consistent with the objectives of The European Network for Cancer Research in Children and Adolescents (ENCCA), as described in 'The First European Interdisciplinary Ewing Sarcoma Research Summit' (53), and provides sufficient evidence to allow discussions on novel therapeutic approaches for the treatment of Ewing's sarcoma family tumors. The most promising results from our study rely on the possibility of using combined regimens that enhance cytotoxic effects, while reducing the undesirable side-effects that are so commonly observed in the ES patient population. Further investigations are needed to consolidate the use of HDIs and bisphosphonates in the therapy of Ewing sarcoma. Understanding the mechanisms by which $\mathrm{NaB}$ and ZA mutually enhance their respective effects is also necessary, in order to potentiate their effects and to develop pharmacophore models for the development of similar drugs that display a broader, but safer, range when used in adjuvant therapy.

\section{Acknowledgements}

The present study was supported by the Rafael Koff Acordi Project (Children's Cancer Institute, Porto Alegre, Brazil) and the Academic Research Hospital Fund 10-0362 (HCPA-FIPE, Porto Alegre, Brazil). We are grateful to Mr. Eduardo Monteiro for his help with elaboration of the images presented herein, OncosinoS and Libbs for the antineoplastic agents and zoledronic acid, respectively, and to all members of the Cancer Research Laboratory.

\section{References}

1. Ricchetti ET, Erol B, Stern J, Russo P, States L and Dormans JP: Lower back pain and mass in a 13 -year-old girl. Clin Orthop Relat Res 430: 248-257, 2005.

2. Carvajal R and Meyers P: Ewing's sarcoma and primitive neuroectodermal family of tumors. Hematol Oncol Clin North Am 19: 501-525, 2005.

3. Karosas AO: Ewing's sarcoma. Am J Health Syst Pharm 67: 1599-1605, 2010

4. Iwamoto Y: Diagnosis and treatment of Ewing's sarcoma. Jpn J Clin Oncol 37: 79-89, 2007.

5. Riggi N and Stamenkovic I: The biology of Ewing sarcoma. Cancer Lett 254: 1-10, 2007. 
6. Jürgens $H$ and Dirksen U: Ewing sarcoma treatment. Eur J Cancer 47: 366-367, 2011.

7. Bernstein M,Kovar H,Paulussen M, et al: Ewing's sarcoma family of tumors: current management. Oncologist 11: 503-519, 2006.

8. Sonnemann J, Dreyer L, Hartwig M, et al: Histone deacetylase inhibitors induce cell death and enhance the apoptosis-inducing activity of TRAIL in Ewing's sarcoma cells. J Cancer Res Clin Oncol 133: 847-858, 2007.

9. Johnson IS, Armstrong JG, Gorman M and Burnett JP Jr: The vinca alkaloids: a new class of oncolytic agents. Cancer Res 23: 1390-1427, 1963

10. Zalupski M and Baker LH: Ifosfamide. J Natl Cancer Inst 80: 556-566, 1988

11. Hande KR: Etoposide: four decades of development of a topoisomerase II inhibitors. Eur J Cancer 34: 1514-1521, 1998.

12. Schimmel KJ, Richel DJ, Van Den Brink RB and Guchelaar HJ: Cardiotoxicity of cytotoxic drugs. Cancer Treat Rev 30: 181-191, 2004.

13. Abujamra AL, Dos Santos MP, Roesler R, Schwartsmann G and Brunetto AL: Histone deacetylase inhibitors: a new perspective for the treatment of leukemia. Leuk Res 34: 687-695, 2010.

14. Marks PA: Histone deacetylase inhibitors: a chemical genetics approach to understanding cellular functions. Biochim Biophys Acta 1799: 717-725, 2010.

15. Marks PA: The clinical development of histone deacetylase inhibitors as targeted anticancer drugs. Expert Opin Investig Drugs 19: 1049-1066, 2010.

16. Hoshino I and Matsubara H: Recent advances in histone deacetylase targeted cancer therapy. Surg Today 40: 809-815, 2010.

17. Pili R, Salumbides B, Zhao M, et al: Phase I study of the histone deacetylase inhibitor entinostat in combination with 13-cis retinoic acid in patients with solid tumours. Br J Cancer 106: 77-84, 2012.

18. dos Santos MP, Schwartsmann G, Roesler R, Brunetto AL and Abujamra AL: Sodium butyrate enhances the cytotoxic effect of antineoplastic drugs in human lymphoblastic T-cells. Leuk Res 33: 218-221, 2009.

19. Clézardin P: Bisphosphonates' antitumor activity: an unravelled side of a multifaceted drug class. Bone 48: 71-79, 2011.

20. Stresing V, Daubiné F, Benzaid I, Mönkkönen H and Clézardin P: Bisphosphonates in cancer therapy. Cancer Lett 257: 16-35, 2007.

21. Odri GA, Dumoucel S, Picarda G, et al: Zoledronic acid as a new adjuvant therapeutic strategy for Ewing's sarcoma patients. Cancer Res 70: 7610-7619, 2010.

22. Zhou Z, Guan H, Duan X and Kleinerman ES: Zoledronic acid inhibits primary bone tumor growth in Ewing sarcoma. Cancer 104: 1713-1720, 2005.

23. de Oliveira MS, Cechim G, Braganhol E, et al: Anti-proliferative effect of the gastrin-release peptide receptor antagonist RC-3095 plus temozolomide in experimental glioblastoma models. J Neurooncol 93: 191-201, 2009.

24. Flores DG, de Farias CB, Leites J, et al: Gastrin-releasing peptide receptors regulate proliferation of C6 glioma cells through a phosphatidylinositol 3-kinase-dependent mechanism. Curr Neurovasc Res 5: 99-105, 2008.

25. Schmidt AL, de Farias CB, Abujamra AL, et al: BDNF and PDE4, but not the GRPR, regulate viability of human medulloblastoma cells. J Mol Neurosci 40: 303-310, 2010.

26. Brunetto de Farias C, Rosemberg DB, Heinen TE, et al: $\mathrm{BDNF} / \mathrm{TrkB}$ content and interaction with gastrin-releasing peptide receptor blockade in colorectal cancer. Oncology 79 430-439, 2010.

27. Cai Z, Chattopadhyay N, Liu WJ, Chan C, Pignol JP and Reilly RM: Optimized digital counting colonies of clonogenic assays using Image J software and customized macros: Comparison with manual counting. Int J Radiat Biol 87: 1135-1146, 2011

28. Chou TC and Talalay P: Quantitative analysis of dose-effect relationships: The combined effects of multiple drugs or enzyme inhibitors. Adv Enz Regul 22: 27-55, 1984.

29. Chou TC: The median-effect principle and the combination index for quantitation of synergism and antagonism. In: Synergism and Antagonism in Chemotherapy. Chou TC and Rideout DC (eds). Academic Press, San Diego, pp61-102, 1991.

30. Gaspar N, Di Giannatale A, Geoerger B, et al: Bone sarcomas: from biology to targeted therapies. Sarcoma 2012: 301975, 2012. doi: $10.1155 / 2012 / 301975$
31. Sakimura R, Tanaka K, Nakatani F, et al: Antitumor effects of histone deacetylase inhibitor on Ewing's family tumors. Int J Cancer 116: 784-792, 2005.

32. Schlottmann S, Erkizan HV, Barber-Rotenberg JS, et al: Acetylation increases EWS-FLI1 DNA binding and transcriptional activity. Front Oncol 2: 1-12, 2012.

33. Li Y, Li X, Fan G, et al: Impairment of p53 acetylation by EWS-Fli1 chimeric protein in Ewing family tumors. Cancer Lett 320: 14-22, 2012

34. Battaglia S, Dumoucel S, Chesneau J, et al: Impact of oncopediatric dosing regimen of zoledronic acid on bone growth: preclinical studies and case report of an osteosarcoma pediatric patient. J Bone Miner Res 26: 2439-2451, 2011.

35. Hirbe AC, Roelofs AJ, Floyd DH, et al: The bisphosphonate zoledronic acid decreases tumor growth in bone in mice with defective osteoclasts. Bone 44: 908-916, 2009.

36. Giuliani N, Pedrazzoni M, Passeri G and Girasole G: Bisphosphonates inhibit IL-6 production by human osteoblast-like cells. Scand J Rheumatol 27: 38-41, 1998.

37. Tenta R, Sourla A, Lembessis P and Koutsilieris M: Bone-related growth factors and zoledronic acid regulate the PTHrP/PTH.1 receptor bioregulation systems in MG-63 human osteosarcoma cells. Anticancer Res 26: 283-291, 2006.

38. Muraro M, Mereuta OM, Saglio F, et al: Interactions between osteosarcoma cell lines and dendritic cells immune function: an in vitro study. Cell Immunol 253: 71-80, 2008.

39. Chauvin C, Philippeau J, Hémont C, et al: Killer dendritic cells link innate and adaptive immunity against established osteosarcoma in rats. Cancer Res 68: 9433-9440, 2008.

40. Sonnemann J, Eckervogt V, Truckenbrod B, Boos J, Winkelmann $\mathrm{W}$ and van Valen F: The bisphosphonate pamidronate is a potent inhibitor of Ewing's sarcoma cell growth in vitro. Anticancer Drugs 14: 767-771, 2003.

41. Siddiqui T, Marsh Rde W, Allegra C, et al: Effective salvage treatment of recurrent Ewing sarcoma utilizing chemotherapy and zoledronic acid. Clin Adv Hematol Oncol 8: 499-504, 2010.

42. Zhao M, Tominaga Y, Ohuchida K, et al: Significance of combination therapy of zoledronic acid and gemcitabine on pancreatic cancer. Cancer Sci 103: 58-66, 2012.

43. Bayrak OF, Aydemir E, Gulluoglu S, et al: The effects of chemotherapeutic agents on differentiated chordoma cells. J Neurosurg Spine 15: 620-624, 2011.

44. Sonnemann J, Bumbul B and Beck JF: Synergistic activity of the histone deacetylase inhibitor suberoylanilide hydroxamic acid and the bisphosphonate zoledronic acid against prostate cancer cells in vitro. Mol Cancer Ther 6: 2976-2984, 2007.

45. Pratap J, Akech J, Wixted JJ, et al: The histone deacetylase inhibitor, vorinostat, reduces tumor growth at the metastatic bone site and associated osteolysis, but promotes normal bone loss. Mol Cancer Ther 9: 3210-3220, 2010.

46. McGee-Lawrence ME and Westendorf JJ: Histone deacetylases in skeletal development and bone mass maintenance. Gene 474: 1-11, 2011.

47. Winter MC, Holen I and Coleman RE: Exploring the anti-tumour activity of bisphosphonates in early breast cancer. Cancer Treat Rev 34: 453-475, 2008.

48. Caraglia M, Santini D, Marra M, Vincenzi B, Tonini G and Budillon A: Emerging anti-cancer molecular mechanisms of aminobisphosphonates. Endocr Relat Cancer 13: 7-26, 2006.

49. Ottewell PD, Woodward JK, Lefley DV, Evans CA, Coleman RE and Holen I: Anticancer mechanisms of doxorubicin and zoledronic acid in breast cancer tumor growth in bone. Mol Cancer Ther 8: 2821-2832, 2009.

50. Frew AJ, Johnstone RW and Bolden JE: Enhancing the apoptotic and therapeutic effects of HDAC inhibitors. Cancer Lett 280: 125-133, 2009.

51. Blanchard F and Chipoy C: Histone deacetylase inhibitors: new drugs for the treatment of inflammatory diseases? Drug Discov Today 10: 197-204, 2005.

52. Minucci S and Pelicci PG: Histone deacetylase inhibitors and the promise of epigenetic (and more) treatments for cancer. Nat Rev Cancer 6: 38-51, 2006.

53. Kovar H, Alonso J, Aman P, et al: The First European Interdisciplinary Ewing Sarcoma Research Summit. Front Oncol 2: $54,2012$. 